\title{
A Vision Based Fish Processing System
}

\author{
Zongjian Zhang, Litao Yu, Jian Zhang and Qiang Wu \\ University of Technology Sydney \\ \{Zongjian.Zhang, Litao.Yu, Jian.Zhang, Qiang.Wu\}@uts.edu.au
}

\begin{abstract}
The digital fish provenance and quality tracking system is essential for the seafood supply chain. As a part of this system, we develop a vision-based fish processing system to automatically perform fish freshness estimation, size measurement and species classification. Under the constrained illumination environment, our system is able to auto-process the fish selection, thus greatly reduce the human labour and bring trust and efficiency to the seafood supply chain from catch to market.
\end{abstract}

\section{INTRODUCTION}

The automatic and streamlined processing with modern AI technologies plays a fundamental role in developing digital seafood agility systems. Consider the following application scenario: at a fish market every weekday in the early morning when the seafood auction begins, buyers can inspect products before deciding which to buy. If the auction system is moved to a digital marketplace, how can buyers be confident in their purchases if they cannot see and evaluate the fish? For these issues, it is necessary to use a digital system to help consumers and suppliers to obtain the verified and trusted information about where the fish was caught, conditions of transportation and ultimately the fish quality attributes. Such a system should incorporate multiple advanced technologies, including wireless communication, embedded device and other automation techniques, to achieve real-time tracking of products along the supply chain, providing comprehensive views in spatial, temporal and other environment domains.

As a part of the digital fish provenance and quality tracking system, one of the objectives is to design an image processing module within an enclosed processing unit. The module should recognize the fish species, estimate the freshness and calibrate the fish size when a fish passed through on a conveyor belt. These functionalities offer the primary information for further processing in digital fish trading. In this paper, we describe the implementation of the prototype, which envisions the development and testing of the system that gives support to digital fish trading in the seafood auction market.

\section{SyStEM IMPLEMENTATION}

\section{A. Hardware settings}

The fish processing system includes two components: frontend image capturer and backend AI server, (see Fig. 1a). On the image capturer side (see right part), a camera is mounted on a height-flexible beam, under which a variety of fish with different sizes and freshness are placed. The image data are then transmitted to the backend AI server equipped with a GPU card for model training. The model is then deployed under a lighting box as an auto-processing unit, which simulates the real application scenario (see left part). In our implementation, the illumination in the lighting box is carefully adjusted to make the illumination condition similar to the environment of data collection, because the change of illumination has very negative impacts on fish freshness estimation.

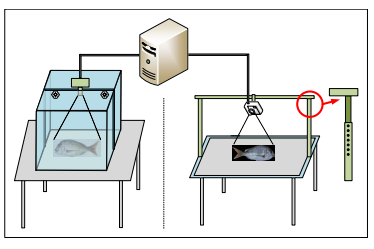

(a) Hardware configuration.

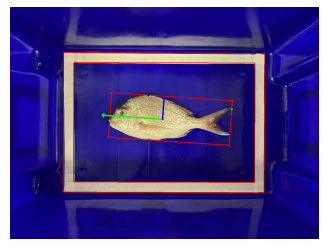

(b) Fish auto-sizing.
Fig. 1: The deployment of our fish processing system.

\section{B. Computation backend}

The computational backend in our system contains three functions: (1) fish species recognition; (2) fish size measurement; and (3) fish freshness estimation.

For the fish species recognition, we used snapper as the test species, according to the requirement from Sydney Fish Market. To achieve this, we collected 2,264 snapper images and 2,711 images of other fish species, to train a binary classifier. Thus, in auto-fish processing, we only focus on snappers as the target species for size measurement and freshness estimation.

The fish size is calculated based on the pixel-length ratio. Within the enclosed a processing unit, the camera is fixed at the top of the lighting box. We first use edge-based contour processing and shape detector to extract the rectangle mark and its corner points, then apply the perspective transformation to obtain the undistorted fish image of a high-angle shot. After that, the a fish detector is used to segment the accurate fish region mask. The orientation of the fish is then computed via PCA, based on all pixel points in the fish mask. Finally, the fish size was calibrated by the pixel-length ratio. In our experiment, the mean absolute error (MAE) of auto-sizing is $0.5 \mathrm{~cm}$.

The fish freshness is defined by three levels: most, medium and least fresh. The model for freshness estimation is essentially a regression model based on the recently proposed EfficientNet [1]. On our collected dataset, we conducted 30 rounds of cross-validation on the total 7,645 images of the 41 snappers for model selection. With the neatly built data environment, the accuracy (after discretization) is $86.1 \%$.

\section{ACKNOWLEDGEMENT}

This project was partially supported by Sydney Fish Market, Australia and Food Agility CRC Ltd under the Australian Government CRC Program. The CRC Program supports industry-led collaborations between industry, researchers and the community.

\section{REFERENCES}

[1] Tan, Mingxing, and Quoc V. Le. Efficientnet: Rethinking model scaling for convolutional neural networks, ICML (2019). 